\title{
Parallel Solvers for Finite-Difference Modeling of Large-Scale, High-Resolution Electromagnetic Problems in MRI
}

\author{
Hua Wang, Adnan Trakic, Feng Liu, Bing Keong Li, Ewald Weber, and Stuart Crozier \\ School of Information Technology and Electric Engineering, The University of Queensland, Brisbane, Qld 4072, Australia
}

Correspondence should be addressed to Stuart Crozier, stuart@itee.uq.edu.au

Received 29 August 2007; Revised 23 January 2008; Accepted 10 April 2008

Recommended by Tamer Ibrahim

\begin{abstract}
With the movement of magnetic resonance imaging (MRI) technology towards higher field (and therefore frequency) systems, the interaction of the fields generated by the system with patients, healthcare workers, and internally within the system is attracting more attention. Due to the complexity of the interactions, computational modeling plays an essential role in the analysis, design, and development of modern MRI systems. As a result of the large computational scale associated with most of the MRI models, numerical schemes that rely on a single computer processing unit often require a significant amount of memory and long computational times, which makes modeling of these problems quite inefficient. This paper presents dedicated message passing interface (MPI), OPENMP parallel computing solvers for finite-difference time-domain (FDTD), and quasistatic finite-difference (QSFD) schemes. The FDTD and QSFD methods have been widely used to model/analyze the induction of electric fields/currents in voxel phantoms and MRI system components at high and low frequencies, respectively. The power of the optimized parallel computing architectures is illustrated by distinct, large-scale field calculation problems and shows significant computational advantages over conventional single processing platforms.
\end{abstract}

Copyright ( $) 2008$ Hua Wang et al. This is an open access article distributed under the Creative Commons Attribution License, which permits unrestricted use, distribution, and reproduction in any medium, provided the original work is properly cited.

\section{INTRODUCTION}

Recent progress in MRI superconducting technology has lead to a considerable increase in human exposure to very large static magnetic fields of up to several Tesla. A typical commercial MRI scanner has a main field strength between 0.5 Tesla and 4 Tesla (in the region of uniformity). However, MRI systems above 4 Tesla, such as 7 Tesla and 9.4 Tesla, are currently used for research [1-3]. Motion of patients and occupational workers through these fields can cause the induction of currents in the body $[4,5]$. In addition, new imaging sequences demand larger amplitudes and switching rates in magnetic field gradients, thus increasing the likelihood of peripheral nerve stimulation (PNS) [69]. When radiofrequency fields are transmitted to excite a spin ensemble during imaging, electromagnetic energy is coupled to the tissue and deposited within, which can cause regional temperature elevations within the patient, thus leading to possible tissue/cell injury $[10,11]$. Apart from interacting with the patient, electromagnetic fields produced by the system also couple to the conducting materials in the MRI system to induce eddy currents that degrade image quality [12]. In practice, it is difficult to measure the unperturbed fields/currents in the human numerical prediction of the induced fields, can then be very useful for the evaluation/design of electromagnetic devices. The aim of this study is to improve the performance of the conventional (quasistatic) finite-difference method, which has been extensively used to model the induction of electric fields/currents in inhomogeneous voxel phantoms of man and animal when exposed to oscillating or static magnetic field sources. The method has the advantages of simplicity and can model any shaped object and various excitation sources.

Numerical computational techniques are indispensable components for analyzing, predicting, and obtaining approximate solutions to the real world problems. The finitedifference time-domain (FDTD) method is well suited to high-frequency electromagnetic analyses due to its simplicity and efficiency in wave modeling and the ability to handle field-material interactions and nonlinear phenomena [13]. In contrast, the quasistatic finite-difference (QSFD) 
method is ideal for linear low-frequency problems with time-harmonic dependency. One example of this is the modeling of the interaction of magnetic fields due to MRI gradients and main superconducting coils with the patient or occupational worker [14-16].

In recent years, we have developed a series of single processor-based finite-difference schemes [14-24], which can be used to simulate a wide range of problems related to MRI and other environments. Our long term aim is to generate a complete temporal model of an MRI system including all the field generating units, passive system components, and an electrical, voxel model of the patient. A better understanding of the complex temporal interaction of the fields within the patient and the system itself can provide useful insight into system design.

Often, numerical modeling of electromagnetic fieldmaterial interactions in MRI requires high-spatial resolution, which imposes a significant computational burden. In solving large-scale-high-resolution (LSHR) models, single processor-based methods are limited and often incapable of managing the required large memory and computational time requirements. Therefore, to improve the performance of these finite difference solvers, it is necessary to further explore computational strategies such as parallelization. Parallel environments such as the message passing interface (MPI), OPENMP, and LAM/MPI have been used in largescale computational problems [25-27]. Due to the geometrical topology of these problems, finite-difference methods and related hybrid algorithms are very adaptable to parallel computing frameworks, in which the computing task is divided and assigned to many processors with distributed or shared memory allocations. This paper outlines MPI and OPENMP parallel computing solvers dedicated to the FDTD and QSFD method, respectively. In the case of the QSFD scheme, a parallelized conjugate gradient technique is applied to solve the sparse matrix equations typical in these situations. A variety of LSHR problems has been investigated using parallel schemes. Compared to previous single processing algorithms, the proposed parallel platforms offer significant advantages in terms of improved numerical convergence and reduced computing time/memory usage. Some of the problems have been solved at high-spatial resolution, which is beyond the capability of single processing methods.

\section{METHODOLOGY}

\subsection{Parallel Cartesian and cylindrical FDTD methods}

Maxwell's equations can be expressed in Cartesian or cylindrical coordinate systems with components of the magnetic $\vec{H}$ and electric field $\vec{E}$ intensity formulated in a general form [28], as given by the following expressions:

$$
\begin{gathered}
\left.H_{A}\right|_{i, j+1 / 2, k+1 / 2} ^{n+1 / 2},\left.C_{h 1} H_{A}\right|_{i, j+1 / 2, k+1 / 2} ^{n}+C_{h 2}\left(\frac{\left.E_{B}\right|_{i, j+1 / 2, k+1} ^{n}-\left.E_{B}\right|_{i, j+1 / 2, k} ^{n}}{\Delta C}\right. \\
\left.\quad-\frac{\left.E_{C}\right|_{i, j+1, k+1 / 2} ^{n}-\left.E_{C}\right|_{i, j, k+1 / 2} ^{n}}{r_{i} \Delta B}\right),
\end{gathered}
$$

$$
\begin{aligned}
& \left.H_{B}\right|_{i+1 / 2, j, k+1 / 2} ^{n+1 / 2} \\
& =\left.C_{h 1} H_{B}\right|_{i+1 / 2, j, k+1 / 2} ^{n} \\
& \quad+C_{h 2}\left(\frac{\left.E_{C}\right|_{i+1, j, k+1 / 2} ^{n}-\left.E_{C}\right|_{i, j, k+1 / 2} ^{n}}{\Delta A}\right. \\
& \left.\quad-\frac{\left.E_{A}\right|_{i+1 / 2, j, k+1} ^{n}-\left.E_{A}\right|_{i+1 / 2, j, k} ^{n}}{\Delta C}\right),
\end{aligned}
$$$$
\left.H_{C}\right|_{i+1 / 2, j+1 / 2, k} ^{n+1 / 2}
$$$$
=\left.C_{h 1} H_{C}\right|_{i+1 / 2, j+1 / 2, k} ^{n}
$$$$
+\frac{C_{h 2}}{r_{i+1 / 2}}\left(\frac{\left.E_{A}\right|_{i+1 / 2, j+1, k} ^{n}-\left.E_{A}\right|_{i+1 / 2, j, k} ^{n}}{\Delta B}\right.
$$$$
\left.-\frac{\left.r_{i+1} E_{B}\right|_{i+1, j+1 / 2, k} ^{n}-\left.r_{i} E_{B}\right|_{i, j+1 / 2, k} ^{n}}{\Delta A}\right),
$$

$$
\begin{aligned}
& \left.E_{A}\right|_{i+1 / 2, j, k} ^{n+1} \\
& =\left.C_{e 1} E_{A}\right|_{i+1 / 2, j, k} ^{n}+\left.\frac{\Delta t}{\varepsilon} J_{A}\right|_{i+1 / 2, j, k} ^{n+1 / 2} \\
& +C_{e 2}\left(\begin{array}{c}
\frac{\left.H_{C}\right|_{i+1 / 2, j+1 / 2, k} ^{n+1 / 2}-\left.H_{C}\right|_{i+1 / 2, j-1 / 2, k} ^{n+1 / 2}}{r_{i+1 / 2} \Delta B} \\
-\frac{\left.H_{B}\right|_{i+1 / 2, j, k+1 / 2} ^{n+1 / 2}-\left.H_{B}\right|_{i+1 / 2, j, k-1 / 2} ^{n+1 / 2}}{\Delta C}
\end{array}\right),
\end{aligned}
$$

$\left.E_{B}\right|_{i, j+1 / 2, k} ^{n+1}$

$$
\begin{aligned}
& =\left.C_{e 1} E_{B}\right|_{i, j+1 / 2, k} ^{n}+\left.\frac{\Delta t}{\varepsilon} J_{B}\right|_{i, j+1 / 2, k} ^{n+1 / 2} \\
& +C_{e 2}\left(\begin{array}{c}
\frac{\left.H_{A}\right|_{i, j+1 / 2, k+1 / 2} ^{n+1 / 2}-\left.H_{A}\right|_{i, j+1 / 2, k-1 / 2} ^{n+1 / 2}}{\Delta C} \\
-\frac{\left.H_{C}\right|_{i+1 / 2, j+1 / 2, k} ^{n+1 / 2}-\left.H_{C}\right|_{i-1 / 2, j+1 / 2, k} ^{n+1 / 2}}{\Delta A}
\end{array}\right),
\end{aligned}
$$

$$
\begin{aligned}
& \left.E_{C}\right|_{i, j, k+1 / 2} ^{n+1} \text {. } \\
& =\left.C_{e 1} E_{C}\right|_{i, j, k+1 / 2} ^{n}+\left.\frac{\Delta t}{\varepsilon} J_{C}\right|_{i, j, k+1 / 2} ^{n+1 / 2} \\
& \quad+\frac{C_{e 2}}{r_{i}}\left(\begin{array}{c}
\frac{\left.r_{i+1 / 2} H_{B}\right|_{i+1 / 2, j, k+1 / 2} ^{n+1 / 2}-\left.r_{i-1 / 2} H_{B}\right|_{i-1 / 2, j, k+1 / 2} ^{n+1 / 2}}{\Delta A} \\
-\frac{\left.H_{A}\right|_{i, j+1 / 2, k+1 / 2} ^{n+1 / 2}-\left.H_{A}\right|_{i, j-1 / 2, k+1 / 2} ^{n+1 / 2}}{\Delta B}
\end{array}\right),
\end{aligned}
$$

where $A=x(r), B=y(\varphi)$, and $C=z$ for Cartesian (cylindrical) coordinates $\Xi=(A, B, C)$. The variable $r_{\xi}(\xi \in$ $\{i, i+1 / 2, i-1 / 2, i+1, i-1\})$ is the radial displacement [in meters] employed in the cylindrical FDTD method. For the Cartesian FDTD method, $r_{\xi}=1$ for all values of $\xi$. In the discrete FDTD computational domain, the dimensions 
of the Yee cell are defined by $\Delta A, \Delta B$, and $\Delta C$. The relative permeability $\mu(\Xi)$, relative permittivity $\varepsilon(\Xi)$, and material conductivity $\sigma(\Xi)$ are assigned at the center of the Yee cell. Parameter $J_{\Xi}$ signifies the impressed current density [in $\mathrm{Am}^{-2}$ ]. The FDTD update coefficients are space-dependent and can be written as follows:

$$
\begin{aligned}
& C_{h 1}=1, \quad C_{h 2}=\frac{\Delta t}{\mu}, \\
& C_{e 1}= \begin{cases}\frac{2 \varepsilon-\sigma \Delta t}{2 \varepsilon+\sigma \Delta t}, & \omega \in \mathrm{RF}, \\
\exp \left(\frac{-\sigma \Delta t}{\varepsilon}\right), & \omega \in \mathrm{LF},\end{cases} \\
& C_{e 2}= \begin{cases}\frac{2 \Delta t}{2 \varepsilon+\sigma \Delta t}, & \omega \in \mathrm{RF}, \\
\frac{1-\exp (-\sigma \Delta t / \varepsilon)}{\sigma}, & \omega \in \mathrm{LF},\end{cases}
\end{aligned}
$$

where $\omega$ is the angular frequency. The abbreviations RF and LF denote radiofrequency and low frequency, respectively. The parameter $\Delta t$ is the FDTD time step, which in general coordinate notation, is limited by the Courant-FriedrichLevy (CFL) stability condition

$$
\Delta t<\frac{k_{t} \sqrt{\mu_{0} \varepsilon}}{\sqrt{(1 / \Delta A)^{2}+\left(1 / r_{M I N} \Delta B\right)^{2}+(1 / \Delta C)^{2}}},
$$

where $\mu_{0}$ and $\varepsilon$ are the permeability and permittivity of free space, respectively, $k_{t}$ is the safety coefficient that ensures numerical stability, and $r_{\mathrm{MIN}}$ is the minimal radial component to be studied (here: $r_{\mathrm{MIN}}=0.5 \Delta r$ ). In the Cartesian coordinate system, $r_{\mathrm{MIN}}=1$. During the updating of the E-field components, the coefficients are linear for the RF case and nonlinear for modeling exponential decay of propagating waves inside good conductors at LF. In LF applications, the conventional FDTD method suffers from prohibitively long computational time [20]. The weakly coupled-Maxwell's equations can be adapted to the lowfrequency regime by downscaling the speed of light constant, which permits the use of larger time steps while maintaining the validity of the CFL stability condition. In our recent study, this modification was accomplished by scaling up the permittivity of free space $\varepsilon_{0}$ by a scaling factor $\alpha: \varepsilon=\varepsilon_{r}\left(\alpha \varepsilon_{0}\right)$. It is clear that when $\alpha=1$, the conventional FDTD methodology for RF applications is obtained. In the cylindrical formulations, the numerical singularity associated with the polar axis $(r=0)$ needs to be considered. A series expansion provides an approximation in the radial direction that satisfies regularity conditions. The combination of cylindrical and Cartesian FDTD methods for both LF and HF applications can be particularly powerful. For instance, the cylindrical FDTD method is particularly suitable for modeling of problems with cylindrical symmetry such as the generation of eddy currents in a conducting cryostat vessel during magnetic field gradient pulsing or the interaction of EM fields produced by radiofrequency resonators and surrounding system materials. The Cartesian

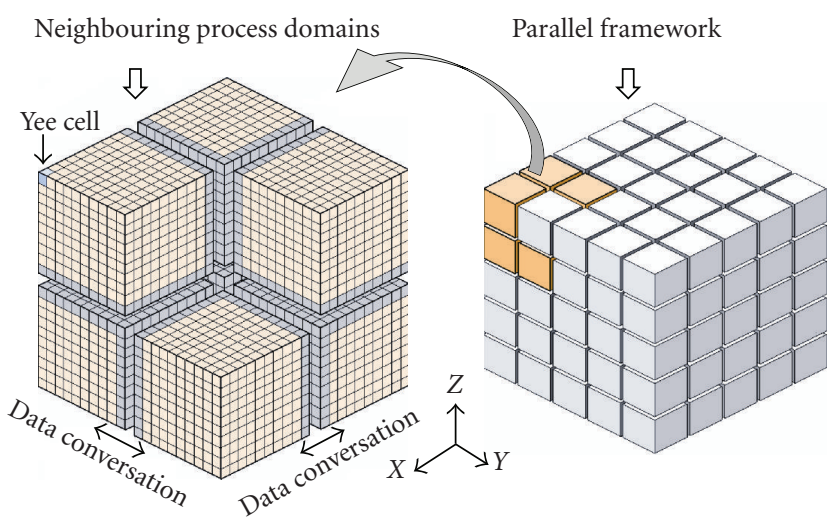

FIGURE 1: Schematic representation of a parallel network with 125 process domains; transmission of field data between neighbouring processes is indicated.

FDTD method allows one to evaluate the electromagnetic wave propagation through tissue-equivalent body models. These FDTD algorithms can be easily adapted to a parallel architecture with the MPI library. With its reputed stability, optional communication routines, and robust compatibility, MPI is considered to be one of the prominent environments to support parallel computing.

In FDTD, the problem space has to be specifically defined before the field calculations commence. In the parallel framework, the computational space is divided into several domains of approximately equal size (see Figure 1) and each domain is managed by one process. All processes run on several processors and execute the same program, while each process operates on memory (computational) domain that is specifically allocated to it. Therefore, mutual access to the memory among the parallel processors is often not straightforward and requires management. With FDTD, additional memory can be assigned to boundary regions of computational subdomains to maintain a smooth transition between neighbouring EM fields on these interfaces. In addition, communication routines can be applied between the neighbouring processes to transmit the E- and $\mathrm{H}$-field components with the consideration of the half time-step difference of these two fields to maintain unified electromagnetic field propagation throughout the entire computational space. Within the MPI frame, a load-balancing scheme is often desired to manage the task distribution in the processing network to improve the computational efficiency.

\subsection{Parallel BiCG method for QSFD scheme}

At low-source frequencies, where the dimensions of the material medium are small compared to the wavelength, the induced fields can be treated quasistatically. According to Faraday's law, the electric field $\vec{E}$ in a conducting sample is induced by a time varying magnetic field $\vec{H}$ due to a source. In terms of electric and magnetic potentials, the total electric field inside the body model can be split into primary field $\vec{E}_{1}$ 
and secondary field $\vec{E}_{2}$, according to

$$
\vec{E}=\vec{E}_{1}+\vec{E}_{2}=-\frac{\partial \vec{A}}{\partial t}-\nabla \Phi
$$

Here, $\vec{A}$ denotes the vector magnetic potential due to the source, and $\Phi$ is the scalar electric potential. The primary electric field $\vec{E}_{1}$ causes a flow of current $\vec{J}_{1}=\sigma \vec{E}_{1}$ in a conductive sample with the conductivity $\sigma$. Any conductivity differences along the path of the current, including the air-tissue interfaces cause nonuniformity of accumulating electric charges, giving rise to scalar potential $\Phi$, the negative gradient of which is the secondary field $\vec{E}_{2}$, which causes a flow of current $\overrightarrow{J_{2}}=\sigma \overrightarrow{E_{2}}$.

Based on our implementation of QSFD method, the computation of the electric fields is given by the governing surface integral equation

$$
\int_{S}\left(\sigma \frac{\partial \vec{A}}{\partial t}\right) \cdot d S=\int_{S}(\sigma \nabla \Phi) \cdot d S
$$

where $S$ represents the surface of the integral region and $\sigma$ is the conductivity of the sample. The governing equation (5) is subject to a boundary condition of the Neumann type:

$$
\frac{\partial \Phi}{\partial n}=\hat{n} \cdot\left(-\frac{\partial \vec{A}}{\partial t}\right)
$$

which indicates that the normal component of the induced eddy current $\vec{J}=\sigma \vec{E}$ on the tissue-air boundary is zero inside the body. To calculate the scalar potential $\Phi$, we divide the computational space into a large number of cubic cells and then (5) is approximated for each elementary cell. After discretization and rearrangement, the scalar potential for cell $(i, j, k)$ can be expressed as

$$
\begin{aligned}
& \Phi_{i, j, k} \\
& =\frac{\sum_{q=0}^{1}\left(\Phi_{i+q, j, k} \sigma_{i+q, j, k}^{a}+\Phi_{i, j+q, k} \sigma_{i, j+q, k}^{a}+\Phi_{i, j, k+q} \sigma_{i, j, k+q}^{a}\right)-f(\vec{A}) h}{\sum_{q=0}^{1}\left(\sigma_{i+q, j, k}^{a}+\sigma_{i, j+q, k}^{a}+\sigma_{i, j, k+q}^{a}\right)},
\end{aligned}
$$

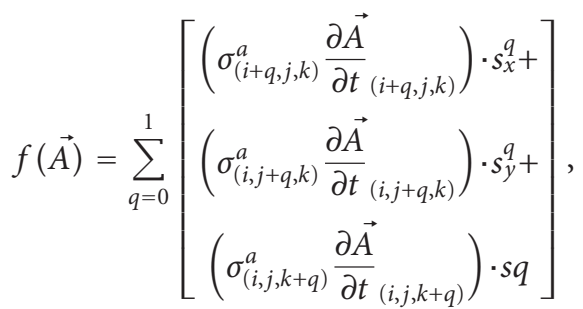

in which subscripts $i, j$, and $k$ indicate the cell indices, subscript $q$ indicates two faces ( 0 or 1$)$ in $x, y, z$ directions, respectively, $s$ is the unit vector normal to the cell faces, $h$ is the cell size, and $\sigma^{a}$ is the local harmonic averaged conductivity. To build the matrix formation, we transform (7) into

$$
\begin{gathered}
\sum_{q=0}^{1}\left(\sigma_{i+q, j, k}^{a}+\sigma_{i, j+q, k}^{a}+\sigma_{i, j, k+q}^{a}\right) \cdot \Phi_{i, j, k}-\sum_{q=0}^{1} \sigma_{i+q, j, k}^{a} \cdot \Phi_{i+q, j, k} \\
-\sum_{q=0}^{1} \sigma_{i, j+q, k}^{a} \cdot \Phi_{i, j+q, k}-\sum_{q=0}^{1} \sigma_{i, j, k+q}^{a} \cdot \Phi_{i, j, k+q}=-f(\vec{A}) h .
\end{gathered}
$$

Equation (8) can then be expressed as a linear relation $\sum_{j=0}^{N} A_{j}^{I} \cdot \Phi_{j}=b_{j}$ in the matrix form of $A \cdot x=b$, where

$$
\begin{aligned}
& A=
\end{aligned}
$$

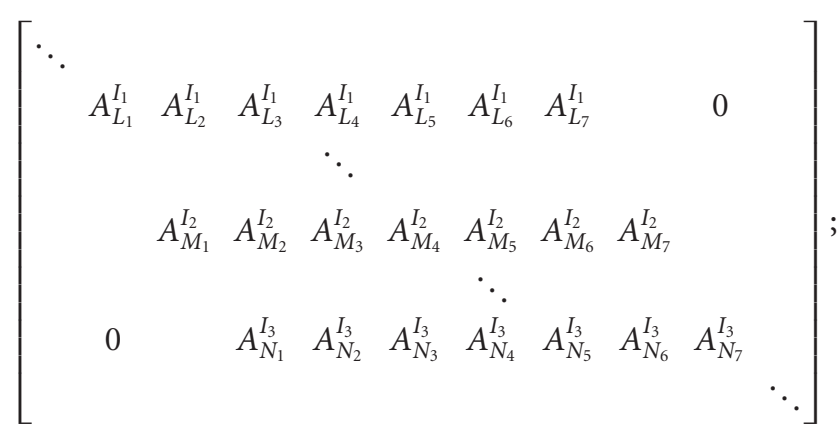

$$
\begin{aligned}
& x=\left[\begin{array}{c}
\Phi_{1} \\
\Phi_{2} \\
\vdots \\
\Phi_{N}
\end{array}\right], \quad b=\left[\begin{array}{c}
b_{1} \\
b_{2} \\
\vdots \\
b_{N}
\end{array}\right] \text {, }
\end{aligned}
$$

where $A$ is the coefficient matrix, $x$ is the scalar potential solution we seek, and $b$ is the known source vector matrix of form $-f(\vec{A}) h$. $N$ is the number of unknowns and appears here as matrix dimension. Assuming the problem scale is $x_{\max } \times y_{\max } \times z_{\max }$, all 3-dimensional $(i, j, k)$ elements are indexed as $I=i \times y_{\max } \times z_{\max }+j \times z_{\max }+k$. Here, $A_{J}^{I}$ represents the linear coefficients between elements $I$ and $J$, and the matrix elements for row $I_{1}$ (see (9)), for instance, are defined as follows:

$$
\begin{gathered}
A_{L_{1}}^{I_{1}}=-\sigma_{i+0, j, k}^{a}, \quad A_{L_{2}}^{I_{1}}=-\sigma_{i, j+0, k}^{a}, \quad A_{L_{3}}^{I_{1}}=-\sigma_{i, j, k+0}^{a}, \\
A_{L_{4}}^{I_{1}}=\sum_{q=0}^{1}\left(\sigma_{i+q, j, k}^{a}+\sigma_{i, j+q, k}^{a}+\sigma_{i, j, k+q}^{a}\right), \\
A_{L_{5}}^{I_{1}}=-\sigma_{i, j, k+1}^{a}, \quad A_{L_{6}}^{I_{1}}=-\sigma_{i, j+1, k}^{a}, \quad A_{L_{7}}^{I_{1}}=-\sigma_{i+1, j, k}^{a},
\end{gathered}
$$

The biconjugate gradient (BICG) method is useful here as the system matrix is nonsymmetric and nonsingular. To solve the linear equations, a row-indexed compact storage method $[29,30]$ has been utilized for the coefficient matrix $A$. With the storage of about twice the number of nonzero matrix elements, compared to other methods requiring as much as three or five times. This scheme also provides sufficient auxiliary information on the matrix and efficient matrix operations using continuous memory. In this way, only nonzero elements have been processed. According to (9), 
coefficients are also assigned to air elements that neighbor elements of the problem space (i.e., those that have nonzero conductivities), thus resulting in sparsity of matrix $A$ in this linear system. BICG requires computing a matrix-vector product and a transpose product. In certain applications, the latter product may be impossible to perform, for example, if the matrix is not formed explicitly and the regular product is only given in operation form, as a function call evaluation. In a parallel computing environment, the two matrix-vector products can be performed simultaneously. However, in a distributed memory environment, there will be extra communication costs associated with one of the two matrixvector products, depending upon the storage scheme for $A$. A duplicate copy of the matrix alleviates this problem, at the cost of doubling the storage requirements for the matrix. A shared memory parallel scheme OPENMP is applied in this case. Without having duplicates on each subprocess, multiple threads compute on one allocated memory. In that way, one can control the number of processing threads to maximize efficiency.

\subsection{Practical applications}

\subsubsection{The RF FDTD application examples}

\section{(a) Rotary phased array head coil-spherical phantom}

To demonstrate the performance of the parallel FDTD method, we investigate a new type of receive-only, 4-element rotary phased array (RPA) head coil that is designed to improve the sensitivity profile at the center of the sample. Shown in Figure 2(a) is the modeled RPA head coil loaded with a spherical phantom. To mimic a simple head model, it was assumed that the FDTD-modeled spherical phantom has a conductivity of $0.616 \mathrm{Sm}^{-1}$ and permittivity of $48.8 \mathrm{Fm}^{-1}$ [31]. Each coil element has a "paddle-like" structure consisting of two main conductors. The main conductors of each coil element will carry equal currents but in opposite directions, as a result, each coil element will produce a plane of maximum sensitivity along the axis of the cylindrical space. That is, the plane of sensitivity of each coil element will cut radially or diametrically through the cylindrical space thus improving the sensitivity at the center of the head coil. The method of moments (MoM) available from a commercial software package FEKO is firstly used to model the RPA head coil and also to ensure that coil elements are decoupled, tuned, and matched to $85 \mathrm{MHz}$. In addition, MoM is used to calculate the initial Huygence equivalent surface sources. Once the initial Huygence equivalent surface sources are obtained and mapped onto FDTD discrete domain, the iteration process commences and is carried out until convergence is achieved. $B_{1}$ fields at the mid-section of the spherical phantom, corresponding to each individual decoupled coil element of the RPA head coil, are calculated and thereafter used for calculating the signal intensity (SI) profiles. The calculated SI profiles are used for comparison with the MR images obtained from the prototype RPA head coil. After obtaining positive simulation results, a prototype RPA head coil was constructed. Shown in Figure 2(b) is the constructed prototype RPA head coil loaded with a spherical phantom. The prototype RPA head coil was tested in a Bruker S200 2T whole-body MRI system, equipped with four receiver channels. Using a MSME pulse sequence with TR $=1000 \mathrm{msec}, \mathrm{TE}=19.3 \mathrm{msec}$, and NEX $=1,4$ axial slices located at the mid-section of the spherical phantom were acquired in parallel by each coil element of the RPA head coil. For cylindrical FDTD modeling, both the conventional single processing and the new parallel FDTD methods were employed and their computational performances compared.

The parallel cylindrical FDTD method was implemented on a 3-server cluster network, each with 2 XEON $3.6 \mathrm{GHz}$ processors and $4 \mathrm{~GB}$ of memory, using an MPI library and managed by a MPICH parallel computing platform. In contrast, the single processing FDTD framework was evaluated on a $3 \mathrm{GHz} / 1 \mathrm{~GB}$ RAM single-CPU machine, which presents identical computing performance on servers. Due to multiuser stimulation, it is very hard to find the best environment to test the single processor FDTD simulation in our server, so we reported the comparison in this way.

\section{(b) Birdcage resonator-human body model}

To further demonstrate the enhanced computing power, we have subjected the parallel Cartesian FDTD algorithm to a very high-spatial resolution of $1 \mathrm{~mm}$. For this purpose, the tissue-equivalent male whole-body models from the Brooks Air force Database [32] at spatial resolutions of $1 \mathrm{~mm}$ and $4 \mathrm{~mm}$ were chosen. The models were subjected to radiofrequency fields generated by a 16-rung whole-body birdcage (volume) resonator operating at $340 \mathrm{MHz}$ (8 Tesla). The dielectric properties of all body-identified tissue types are frequency scaled and kept constant at the designated frequency [33]. At high frequencies, it is impossible to have a global uniform flip angle in the whole imaging slice, field focusing scheme is therefore implemented. The input power of the birdcage resonator is numerically adjusted (a scaling is done to the fields) to provide an averaged 90-degree flip angle in the subregion of the abdomen and chest, such as the heart. The space domain enclosing the birdcage resonator, perfectly matched layers (PML) and the human phantom was modeled with $626 \times 380 \times 1918$ cells at $1 \mathrm{~mm}$ and $186 \times 135 \times 510$ cells at $4 \mathrm{~mm}$ resolution. After the steady-state is obtained, the specific absorption rate (SAR) is computed using the following equation:

$$
\operatorname{SAR}(\Xi)=\frac{\sigma(\Xi)|E(\Xi)|^{2}}{2 \rho(\Xi)},
$$

where $\Xi=(A, B, C)$ is the coordinate of the voxel in designated coordinate system, $\sigma$ is the conductivity of tissue $\left(\mathrm{Sm}^{-1}\right), E$ is the peak recorded electric field intensity $\left(\mathrm{Vm}^{-1}\right)$, and $\rho$ is the specific mass density of tissue $\left(\mathrm{kgm}^{-1}\right)$.

The models were implemented on an SGI highperformance computer (HPC) with 10 computing nodes, where each node is equipped with 2 Itanium 2nd CPU $1.5 \mathrm{GHz}$ and $4 \mathrm{~GB}$ of memory. The $1 \mathrm{~mm}-$ and $4 \mathrm{~mm}-$ resolution cases were performed using 10 and 5 computing nodes, respectively. 


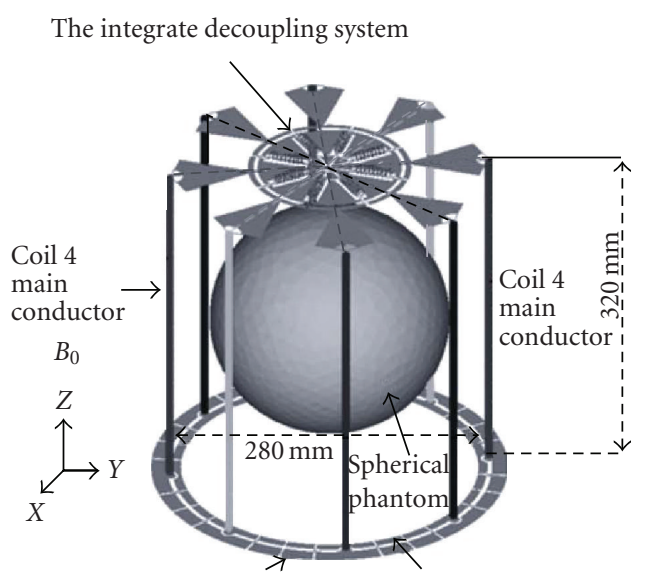

The two circular conductor bases

$$
\begin{array}{cr}
\text { Coil } 1 & \text { Coil } 3 \\
- \text { Coil } 2 & \text { Coil } 4
\end{array}
$$

(a)
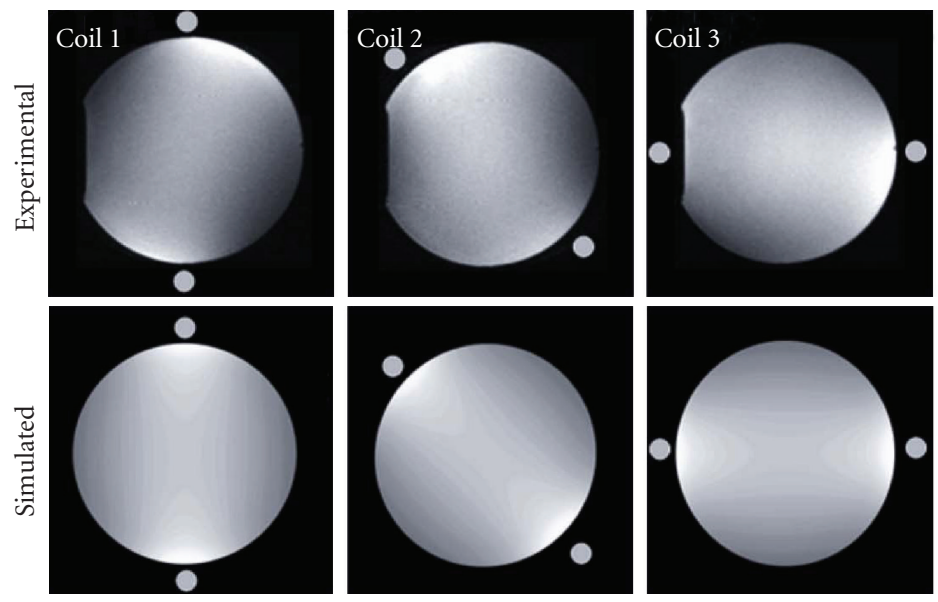

(c)

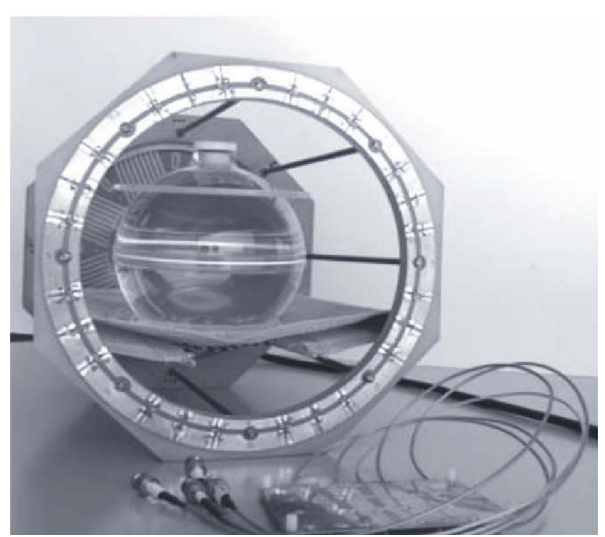

(b)
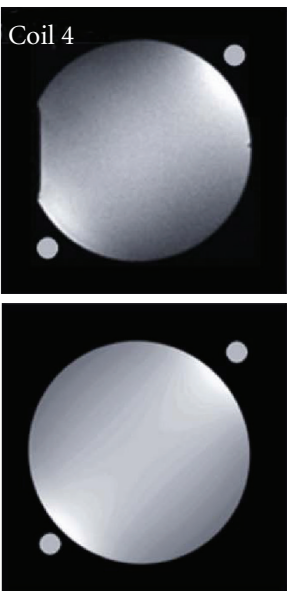

FIGURE 2: (a) FDTD model setup of the RPA head coil and spherical phantom, (b) prototype RPA coil and (c) Signal intensities corresponding to each coil element evaluated with the FDTD method and obtained experimentally during MR imaging.

\subsubsection{The QSFD application example}

When MRI operators (radiographers or MR technicians) attend anxious, sedated, or intubated patients during an MRI exam, they can be repeatedly exposed to switched magnetic field gradients near the bore entrance at the imager end [5]. In this study, we use the QSFD-BiCG method to evaluate the exposure of an MRI occupational worker in the vicinity of the MRI machine. In particular, the computational performance of the single processing versus parallel QSFD method is assessed.

\section{(a) Body models}

Anatomically, accurate whole-body voxel models of male (BROOK) and female (NAOMI, [33]) are used to represent the occupational workers. The female model was engaged at different voxel resolutions to study the computational performances of the single processing and parallel QSFD method. The scales of the female model in Cartesian dimensions were $277 \times 148 \times 871,147 \times 62 \times 396,98 \times$ $42 \times 264$, and $74 \times 31 \times 198$ (in $x, z$, and $y$ ) at model resolutions of $2 \mathrm{~mm}, 4 \mathrm{~mm}, 6 \mathrm{~mm}$, and $8 \mathrm{~mm}$, respectively. In a computationally extreme case, the male model was studied at an ultra-high resolution of only $1 \mathrm{~mm}$, whereby the scales of the model in Cartesian dimensions were $586 \times 340 \times$ 1878 . The experimentally-determined conductivity values by Gabriel et al. [34] of some forty body-identified tissue types were aptly scaled to the frequency of interest and assigned to the appropriate body voxels.

\section{(b) Gradient coils}

An actively-shielded whole-body assembly, consisting of the $x-, y$-, and $z$-axis gradient coil is used to evaluate the 
electric fields and current densities induced in the worker during gradient switching $[12,35]$. For mere demonstration purposes, all three gradient coils have been designed to have approximately same axial length of $\sim 1.4 \mathrm{~m}$, yet to remain radially separated, that is, the six coil layers (primary and secondary) are allocated to different radii assuming a layer thickness (including former, etc.) of $5 \mathrm{~mm}$. The length of the gradient set is assumed to be of the same length as the imager including the cryostat vessel. Table 1 lists some important parameters while Figure 3(a) illustrates the geometries of the three gradient coils (note that the $y$-gradient coil is identical to the $x$-gradient coil when rotated by 90 degrees). It is assumed that each gradient coil generates a normalized gradient field strength of $1 \mathrm{mT} / \mathrm{m}$ in the working volume, and that the gradient coil currents are pulsed trapezoidally at the frequency of $1 \mathrm{kHz}$ and 100 microseconds rise time.

\section{(c) Computational setup}

Figure 4(a) illustrates a sketch of the position and orientation for both body models near the gradient set end outside the MRI machine. An iterative method based on the SOR algorithm [14-16] and a new parallel BiCG method developed in this study are engaged to compare the numerical convergence and computing performance. All numerical modeling with the parallel QSFD-BiCG method were performed on the aforementioned SGI HPC with 10 computing nodes. Ten subthreads are assigned by the OPENMP scheme. The single processor simulations were performed on the same computing platform with only one computing node assigned.

\section{RESULTS AND DISCUSSION}

In our experience, numerical studies that involve highresolution $(\sim 1 \mathrm{~mm})$ models of human head, pelvis, or other parts of the body have been published. There is, however, no (or lack of) work that involves high-resolution wholebody models, such as $1 \mathrm{~mm}$. This work reports interesting results involving a $1 \mathrm{~mm}$ resolution whole-body model, which previously was not possible on single CPU platforms.

\subsection{Example RF FDTD applications}

\section{(a) Current carrying loop-spherical phantom}

Shown in Figure 2(c) are the SI profiles obtained using the MoM/FDTD method and experimentally acquired MR images of the spherical phantom using the prototype RPA head coil. It can be observed that both numerical and experimental results agree well and that the sensitivity at the center of the spherical phantom, as anticipated, has been improved using the RPA head coil. Both the single processing and parallel FDTD methods require around $100 \mathrm{MB}$ RAM and yield identical results. The computing time using the nonparallelized FDTD method was around 182 seconds. In contrast, the parallel FDTD method is around six times faster than the conventional scheme.

\section{(b) Birdcage resonator-human body model}

Figure 5(a) shows the model setup involving body and the birdcage resonator. Figure 5(b) compares the specific absorption rate (SAR) at the body-model resolution of $1 \mathrm{~mm}$ and $4 \mathrm{~mm}$. Based on the current hardware settings, the whole-body model at $1 \mathrm{~mm}$ spatial resolution required approximately $28 \mathrm{~GB}$ of memory and 20 hours of computing time for 6000 FDTD iterations to get converged results. The low-resolution simulation $(4 \mathrm{~mm})$ required around $500 \mathrm{MB}$ of memory and 1.8 hours of computing time. Compared to spatial SAR distribution at $4 \mathrm{~mm}$, the $1 \mathrm{~mm}$ resolution model provides us with clearly more detail and better tissue specificity.

In terms of problem scale, the $1 \mathrm{~mm}$ resolution case is around $4^{3}=64$ times larger than the $4 \mathrm{~mm}$ simulation. To each processing node involved, the time consumption for $1 \mathrm{~mm}$ processing is only about 11 times larger than for the lower-resolution case, which illustrates the highercomputational efficiency of the parallel scheme.

The single processing FDTD routine at $1 \mathrm{~mm}$ resolution could not be implemented due to the very large amount of memory required. In this sense, the parallelism cannot only improve the computational efficiency of FDTD, but enable it to solve those problems that conventional FDTD cannot. In the parallel FDTD computing framework, data communication occupies much less time than the computation of E-H field components. Thus, implementations with extensive task distribution can improve computational efficiency, but the additional communication overhead may affect the overall performance. In theory, the time complexity is inversely proportional to the number of parallel threads, provided there is enough random access memory (RAM) and CPU processing resources. With the inevitable communication overhead imposed on the individual processes, however, execution efficiency is often significantly diminished. A loadbalancing scheme is applied in the parallelization to reduce the latency time between subprocesses which eventually alleviates both the data conflicts and iterative latency in the data transmission. This parallel FDTD scheme has remarkable computing advantages over conventional single processing methods, which could be also seen in many other parallel computing scenarios though in different applications [25-27].

\subsection{Example QSFD application}

Figure 4(a) depicts the typical posture of the radiographer on the side of the patient bed, which was used in the QSFD computation. Figure 4(b) represents the spatial distributions of the in situ electric field and current density-induced in the female model during the exposure to the combination of switched gradient coils. Figure 4(c) shows a comparison of the induced average current density versus superior-inferior axis obtained using the parallel BiCG and SOR.

Although the large-scale model used is very inhomogeneous and the source field varies dramatically in the spatial domain, both methods provided remarkably similar simulation results in terms of resulting field magnitudes and 
TABLE 1: Geometrical properties of gradient coils.

\begin{tabular}{lccc}
\hline Parameters & $X$-gradient & $Y$-gradient & $Z$-gradient \\
\hline 1st layer $-Z[\mathrm{~m}]$ & 1.18 & 1.20 & 1.29 \\
2nd layer $-Z[\mathrm{~m}]$ & 1.37 & 1.40 & 1.40 \\
1st layer $-R[\mathrm{~m}]$ & 0.31 & 0.32 & 0.33 \\
2nd layer $-R[\mathrm{~m}]$ & 0.36 & 0.37 & 0.39 \\
DSV: $r[\mathrm{~m}] \times z[\mathrm{~m}]$ & $0.42 \times 0.42$ & $0.42 \times 0.42$ & $0.50 \times 0.56$ \\
\hline
\end{tabular}

Note: $Z$ denotes axial length and $R$ denotes the radius of the primary and secondary gradient coil layers. The DSV size is given as the region where the gradient field is uniform to $5 \%$ peak-peak and is expressed as diameter by length in meters.
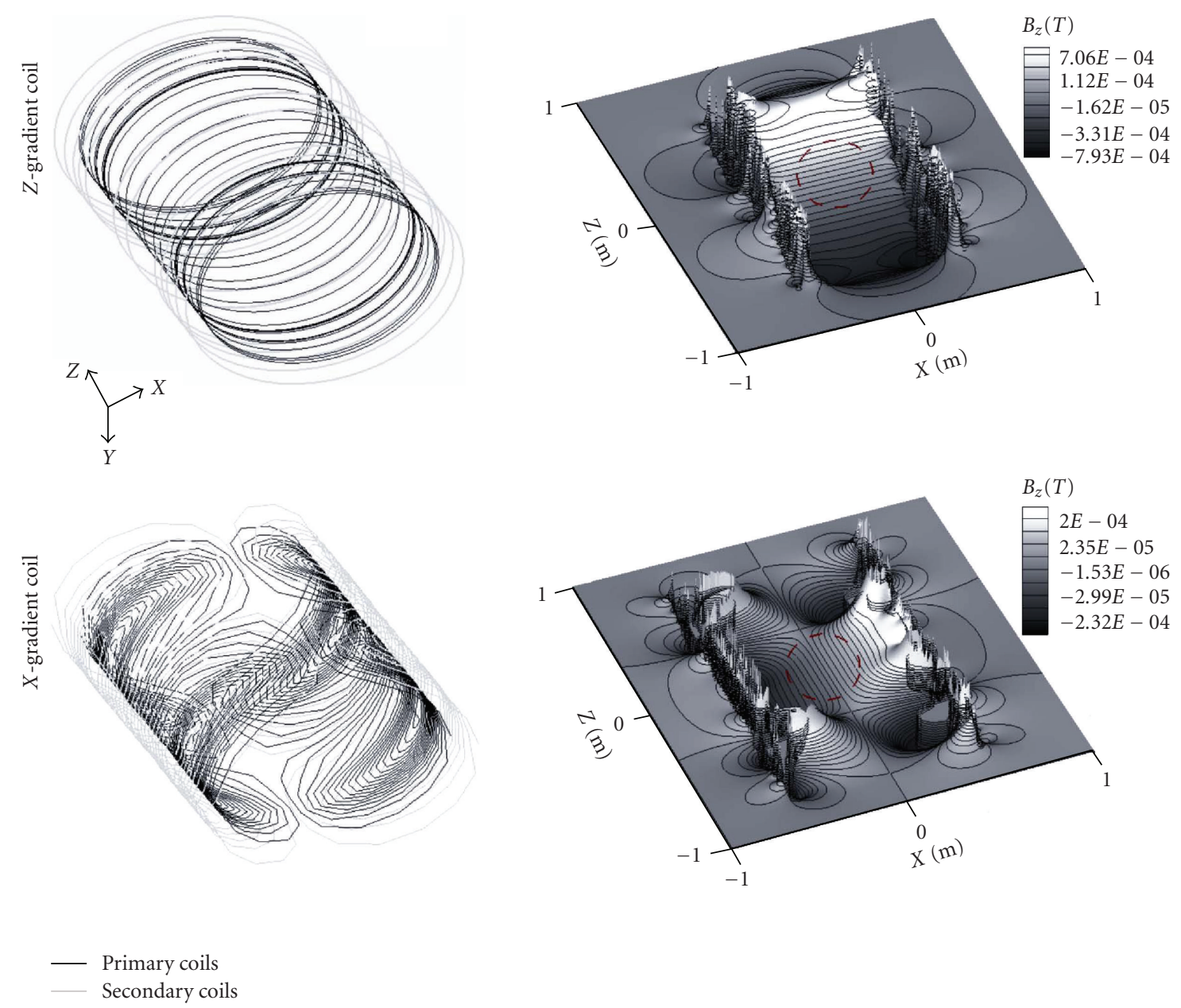

(a)

(b)

FIgURE 3: (a) Three-dimensional plot of transverse and longitudinal gradient coil geometry (the primary and secondary windings are indicated in black and grey, resp.) and (b) corresponding spatial gradients of $B_{z}$. DSV is the diameter spherical volume.

spatial distributions, with less than $1 \%$ relative difference. According to the convergence performance results detailed in Table 2, the QSFD-BiCG method clearly outperforms the SOR-based QSFD technique at all nominated resolutions of the female body model.

According to Table 2, the coefficient matrix dimension grows dramatically up to 8 million elements with $2 \mathrm{~mm}$ resolution, which brings about tremendous computational burdens. Via the computing parallelization, both SOR and
BiCG methods have been improved, while the latter shows better convergence performance.

Using the $1 \mathrm{~mm}$ resolution male body model, the parallel QSFD-BiCG method took around 23 hours and $28 \mathrm{~GB}$ of RAM to evaluate in situ electric fields/currents. The number of elements in matrix $A$ was $\sim 1.4 \cdot 10^{17}$. In contrast, the single processing equivalent was unable to perform this particular simulation due to immense memory requirements. This example demonstrates one of the clear advantages of the 


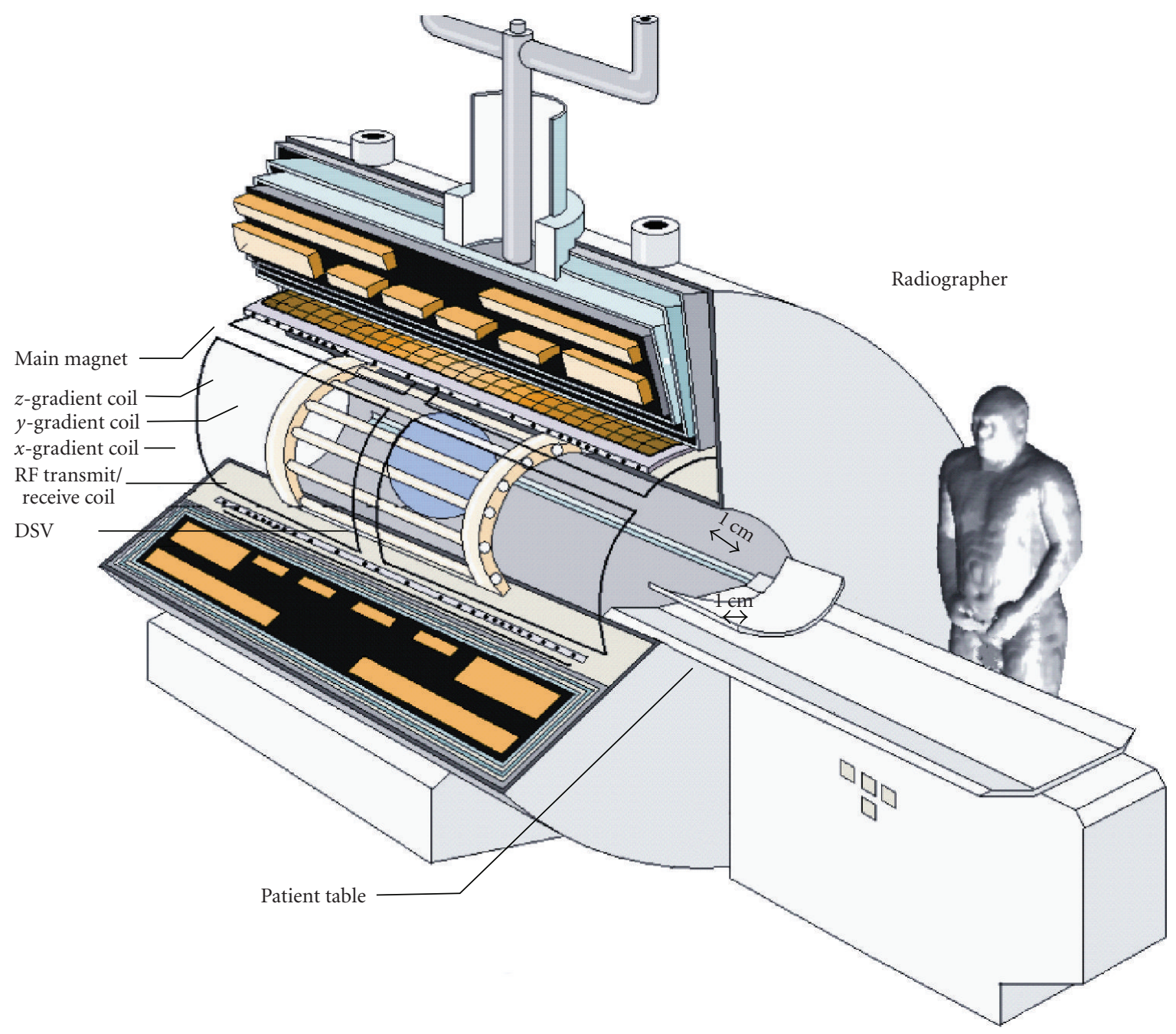

(a)

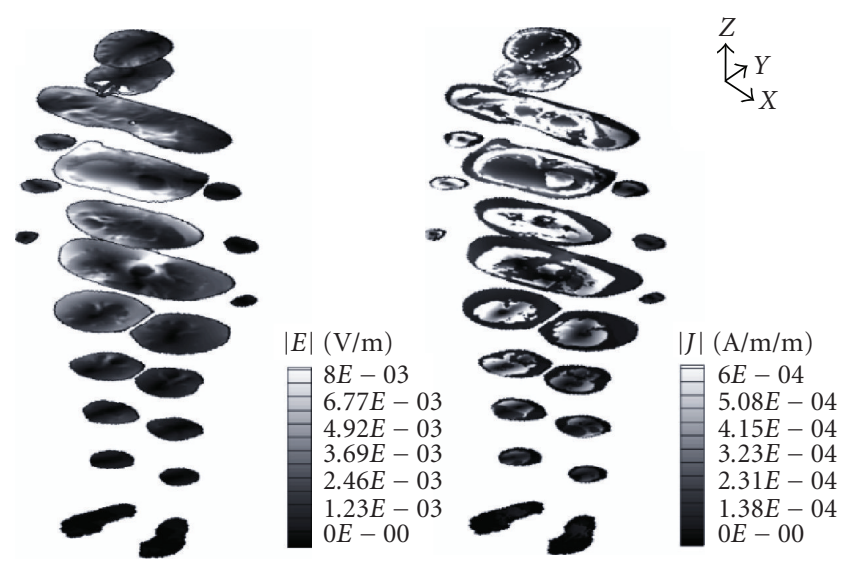

(b)

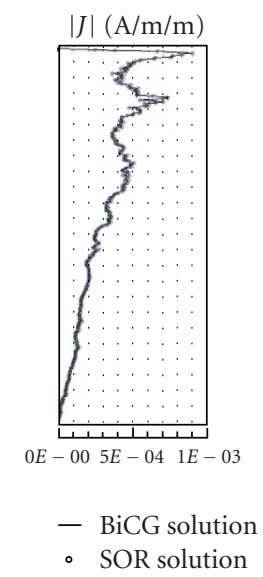

(c)

FIgUre 4: (a) Typical body posture near the imager where gradient coils are assumed to be pulsed as described in the text, (b) axial slices illustrating the spatial distributions of induced electric field and current density and (c) comparison of the average current density along the superior-inferior axis between the QSFD-BiCG and QSFD-SOR methods indicating less than 1\% of relative deviation. 


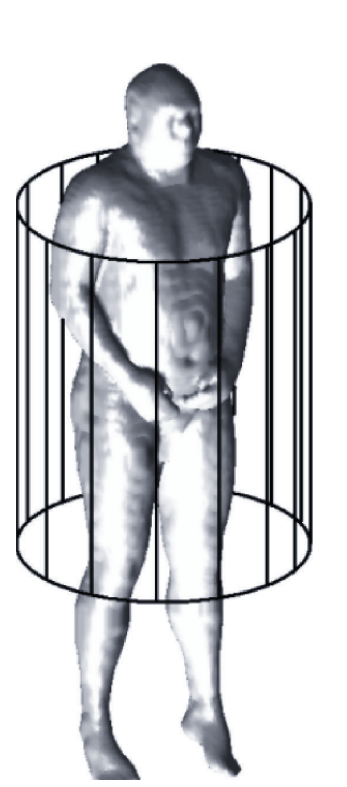

(a)

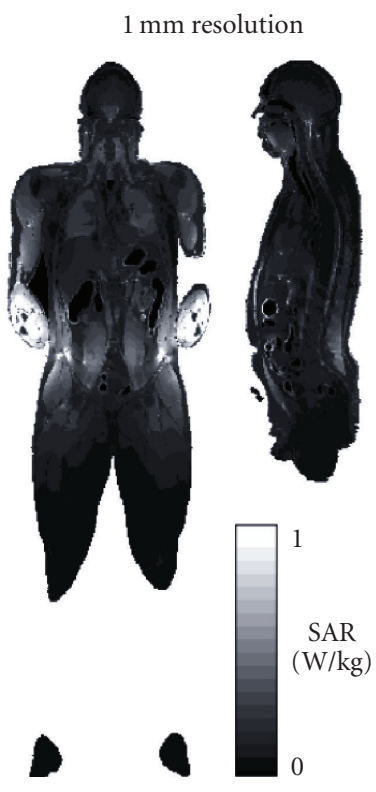

(b)

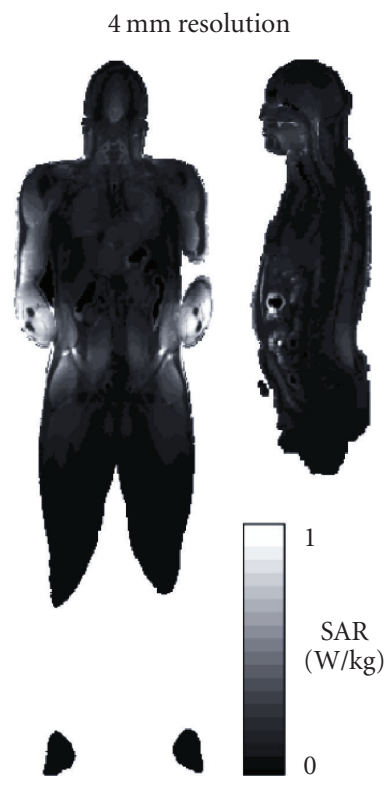

FIGURE 5: (a) Model setup involving the whole-body birdcage resonator and the body model, (b) coronal and sagittal profiles of in situ SAR spatial distributions in the male Brooks Airforce model (BROOK) at $1 \mathrm{~mm}$ and $4 \mathrm{~mm}$ voxel resolution.

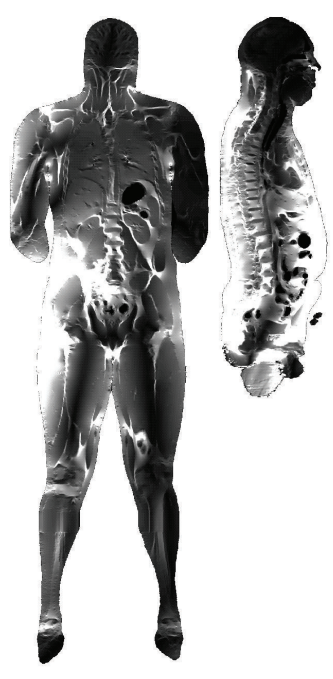

(a)
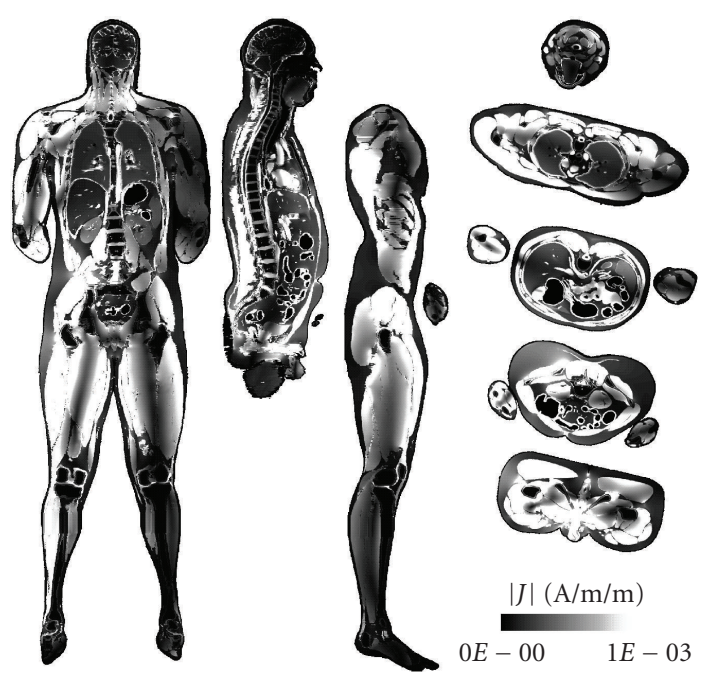

(b)

FIGURE 6: Coronal, sagittal and axial distributions of (a) electric field and (b) current density induced in the 1mm-resolution male body model during gradient pulsing. Figure 5(a) illustrates the body posture of the model relative to the gradient set.

TABLE 2: Computing performance.

\begin{tabular}{lcccc}
\hline Property of coefficient matrix $A$ & \multicolumn{3}{c}{ Problem resolution } \\
& $8 \mathrm{~mm}$ & $6 \mathrm{~mm}$ & $4 \mathrm{~mm}$ & $2 \mathrm{~mm}$ \\
\hline Scale $(n \times n)$ & $153699 \times 153699$ & $350786 \times 350786$ & $1134416 \times 1134416$ & $8670828 \times 8670828$ \\
Nonzero elements & 884439 & 2109038 & 7150900 & 57488850 \\
\hline Convergence performance & & & & \\
\hline Parallelized SOR & 2.5 minutes & 14 minutes & 36 minutes & 31 hours \\
Single-CPU BiCG & 10 seconds & 39 seconds & 3 minutes & 1.8 hours \\
Parallelized BiCG & 7 seconds & 23 seconds & 1.5 minutes & 50 minutes \\
\hline
\end{tabular}


parallel QSFD computing platform over its conventional single processor-based counterpart. Figures 6(a) and 6(b) illustrate very fine details of induced electric field and current density distributions for the male body model, respectively. According to the simulation results, the largest electric fields and current densities are located in the frontal left region of the body, as this is the part of the body that is closest to the gradient coil conductors. With well-conditioned band diagonal sparse coefficient matrices, the proposed parallel QSFD-BiCG scheme demonstrates robust performance in the handling of low-frequency electromagnetic fields problems. The BiCG method also shows efficient memory usage, as it only consumes about $1 / 3$ of memory compared to the standard SOR scheme.

This level of high-resolution analyses is imperative and significant for those interested in a variety of numerical problems such as but not limited to: thermodynamic modeling involving a fine vascular structure, cardiac electric field propagation, hyperthermia, eddy current modeling, occupational exposure of workers to electromagnetic fields, and so forth. Most of these and other related problems would benefit from employing the whole body in the computational process, as this represents a more realistic physical scenario.

\section{CONCLUSION}

Computationally intensive numerical software has become necessary to handle the increasing complexity of electromagnetic field problems in MRI, particularly at high-field strengths. In this work, high-performance finite-difference solvers architected within a parallel framework have been presented. The potential of the optimized parallel scheme has been demonstrated in typical MRI applications. The case studies indicate that the power of the software can enable straightforward adaptation to applications involving optimization of system components.

\section{ACKNOWLEDGMENTS}

The financial support for this project from the Australian Research Council and The Health and Safety Executive (UK) (the project managed by MCL, UK) is gratefully acknowledged.

\section{REFERENCES}

[1] A. K. Andriola Silva, É. L. Silva, E. S. T. Egito, and A. S. Carriço, "Safety concerns related to magnetic field exposure," Radiation and Environmental Biophysics, vol. 45, no. 4, pp. 245-252, 2006.

[2] J. F. Schenck, "Safety of strong, static magnetic fields," Journal of Magnetic Resonance Imaging, vol. 12, no. 1, pp. 2-19, 2000.

[3] P. A. Gowland, "Present and future magnetic resonance sources of exposure to static fields," Progress in Biophysics and Molecular Biology, vol. 87, no. 2-3, pp. 175-183, 2005.

[4] S. Crozier and F. Liu, "Numerical evaluation of the fields caused by body motion in or near high-field MRI scanners," Progress in Biophysics and Molecular Biology, vol. 87, no. 2-3, pp. 267-278, 2005.
[5] "Assessment of electromagnetic fields around magnetic resonance imaging (MRI) equipment," Tech. Rep. RR570, Health and Safety Executive, London, UK, 2007.

[6] F. X. Hebrank, "SAFE model—a new method for predicting peripheral nerve stimulation in MRI," in Proceedings of the 8th Annual Meeting of the International Society for Magnetic Resonance in Medicine (ISMRM '00), vol. 8, pp. 2007-2013, Denver, Colo, USA, April 2000.

[7] D. J. Schaefer, J. D. Bourland, and J. A. Nyenhuis, "Review of patient safety in time-varying gradient fields," Journal of Magnetic Resonance Imaging, vol. 12, no. 1, pp. 20-29, 2000.

[8] S. C. Faber, A. Hoffmann, C. Ruedig, and M. Reiser, "MRIinduced stimulation of peripheral nerves: dependency of stimulation threshold on patient positioning," Magnetic Resonance Imaging, vol. 21, no. 7, pp. 715-724, 2003.

[9] J. D. Bourland, J. A. Nyenhuis, and D. J. Schaefer, "Physiologic effects of intense MR imaging gradient fields," Neuroimaging Clinics of North America, vol. 9, no. 2, pp. 363-377, 1999.

[10] C. M. Collins, S. Li, and M. B. Smith, "SAR and $B_{1}$ field distributions in a heterogeneous human head model within a birdcage coil," Magnetic Resonance in Medicine, vol. 40, no. 6, pp. 847-856, 1998.

[11] T. S. Ibrahim, R. Lee, B. A. Baertlein, Y. Yu, and P.-M. L. Robitaille, "Computational analysis of the high pass birdcage resonator: finite difference time domain solutions for highfield MRI," Magnetic Resonance Imaging, vol. 18, no. 7, pp. 835-856, 2000.

[12] A. Trakic, F. Liu, H. Sanchez Lopez, H. Wang, and S. Crozier, "Longitudinal gradient coil optimization in the presence of transient eddy currents," Magnetic Resonance in Medicine, vol. 57, no. 6, pp. 1119-1130, 2007.

[13] A. Taflove, Computational Electrodynamics: The FiniteDifference Time-Domain Method, Artech House, Boston, Mass, USA, 1995.

[14] F. Liu, H. W. Zhao, and S. Crozier, "On the induced electric field gradients in the human body for magnetic stimulation by gradient coils in MRI," IEEE Transactions on Biomedical Engineering, vol. 50, no. 7, pp. 804-815, 2003.

[15] S. Crozier and F. Liu, "Numerical evaluation of the fields caused by body motion in or near high-field MRI scanners," Progress in Biophysics and Molecular Biology, vol. 87, no. 2-3, pp. 267-278, 2005.

[16] F. Liu, H. Zhao, and S. Crozier, "Calculation of electric fields induced by body and head motion in high-field MRI," Journal of Magnetic Resonance, vol. 161, no. 1, pp. 99-107, 2003.

[17] F. Liu, S. Crozier, H. Zhao, and B. Lawrence, "Finite-difference time-domain-based studies of MRI pulsed field gradientinduced eddy currents inside the human body," Concepts in Magnetic Resonance Part B, vol. 15, no. 1, pp. 26-36, 2002.

[18] H. Zhao, S. Crozier, and F. Liu, "Finite difference time domain (FDTD) method for modeling the effect of switched gradients on the human body in MRI," Magnetic Resonance in Medicine, vol. 48, no. 6, pp. 1037-1042, 2002.

[19] H. Zhao, S. Crozier, and F. Liu, "A high definition, finite difference time domain method," Applied Mathematical Modelling, vol. 27, no. 5, pp. 409-419, 2003.

[20] F. Liu and S. Crozier, "A distributed equivalent magnetic current based FDTD method for the calculation of E-fields induced by gradient coils," Journal of Magnetic Resonance, vol. 169, no. 2, pp. 323-327, 2004.

[21] Q. Wei, F. Liu, L. Xia, and S. Crozier, "An objectoriented designed finite-difference time-domain simulator for 
electromagnetic analysis and design in MRI-applications to high field analyses," Journal of Magnetic Resonance, vol. 172, no. 2, pp. 222-230, 2005.

[22] F. Liu and S. Crozier, "An FDTD model for calculation of gradient-induced eddy currents in MRI system," IEEE Transactions on Applied Superconductivity, vol. 14, no. 3, pp. 1883-1889, 2004.

[23] A. Trakic, H. Wang, F. Liu, H. S. López, and S. Crozier, "Analysis of transient eddy currents in MRI using a cylindrical FDTD method," IEEE Transactions on Applied Superconductivity, vol. 16, no. 3, pp. 1924-1936, 2006.

[24] F. Liu, B. L. Beck, B. Xu, J. R. Fitzsimmons, S. J. Blackband, and S. Crozier, "Numerical modeling of 11.1T MRI of a human head using a MoM/FDTD method," Concepts in Magnetic Resonance Part B, vol. 24, no. 1, pp. 28-38, 2005.

[25] C. Guiffaut and K. Mahdjoubi, "A parallel FDTD algorithm using the MPI library," IEEE Antennas and Propagation Magazine, vol. 43, no. 2, pp. 94-103, 2001.

[26] S. D. Gedney, "Finite-difference time-domain analysis of microwave circuit devices on high performance vector/parallel computers," IEEE Transactions on Microwave Theory and Techniques, vol. 43, no. 10, pp. 2510-2514, 1995.

[27] K. Taguchi, M. Uchiya, T. Kashiwa, K. Hirayama, H. Kuribayashi, and S. Komatsu, "FDTD large-scale parallel supercomputing and its application to the analysis of radiation characteristics of an antenna mounted on a vehicle," International Journal of RF and Microwave Computer-Aided Engineering, vol. 14, no. 3, pp. 253-261, 2004.

[28] K. S. Yee, "Numerical solution of initial boundary value problems involving Maxwell's equations in isotropic media," IEEE Transactions on Antennas and Propagation, vol. 14, no. 5, pp. 302-307, 1996.

[29] W. H. Press, S. A. Teukolsky, W. T. Vetterling, and B. P. Flannery, Numerical Recipes in C: The Art of Scientific Computing, Cambridge University Press, Cambridge, UK, 1992.

[30] PCGPAK User's Guide, Scientific Computing Associates, New Haven, Conn, USA.

[31] F. Liu and S. Crozier, "Electromagnetic fields inside a lossy, multilayered spherical head phantom excited by MRI coils: models and methods," Physics in Medicine and Biology, vol. 49, no. 10, pp. 1835-1851, 2004.

[32] Human male voxel phantom BROOK, "Brooks Air force Database," 2004, http://www.brooks.af.mil/.

[33] P. Dimbylow, "Development of the female voxel phantom, NAOMI, and its application to calculations of induced current densities and electric fields from applied low frequency magnetic and electric fields," Physics in Medicine and Biology, vol. 50, no. 6, pp. 1047-1070, 2005.

[34] C. Gabriel, S. Gabriel, and E. Corthout, "The dielectric properties of biological tissues-I: literature survey," Physics in Medicine and Biology, vol. 41, no. 11, pp. 2231-2249, 1996.

[35] S. Crozier, L. K. Forbes, and D. M. Doddrell, "The design of transverse gradient coils of restricted length by simulated annealing," Journal of Magnetic Resonance A, vol. 107, no. 1, pp. 126-128, 1994. 

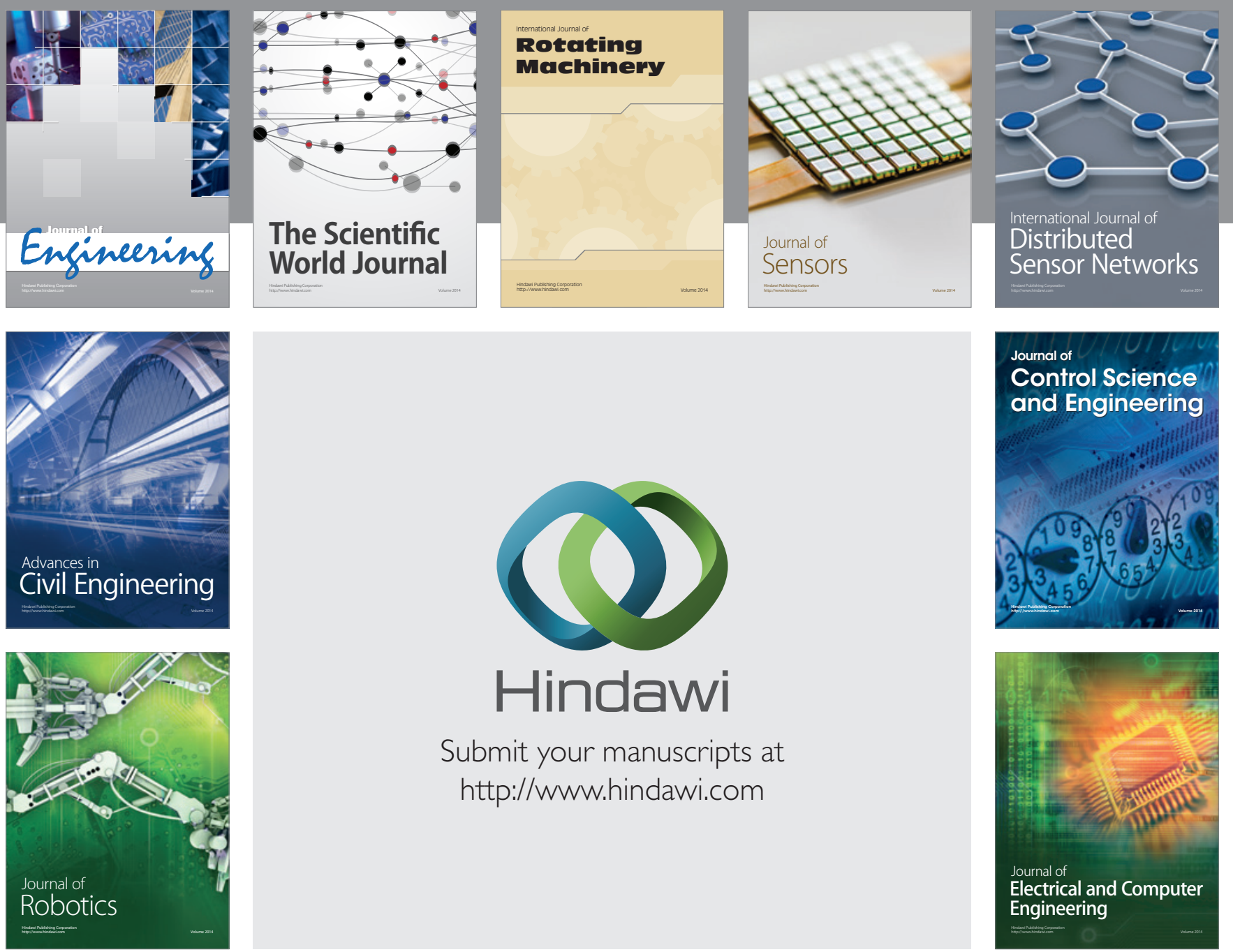

Submit your manuscripts at

http://www.hindawi.com
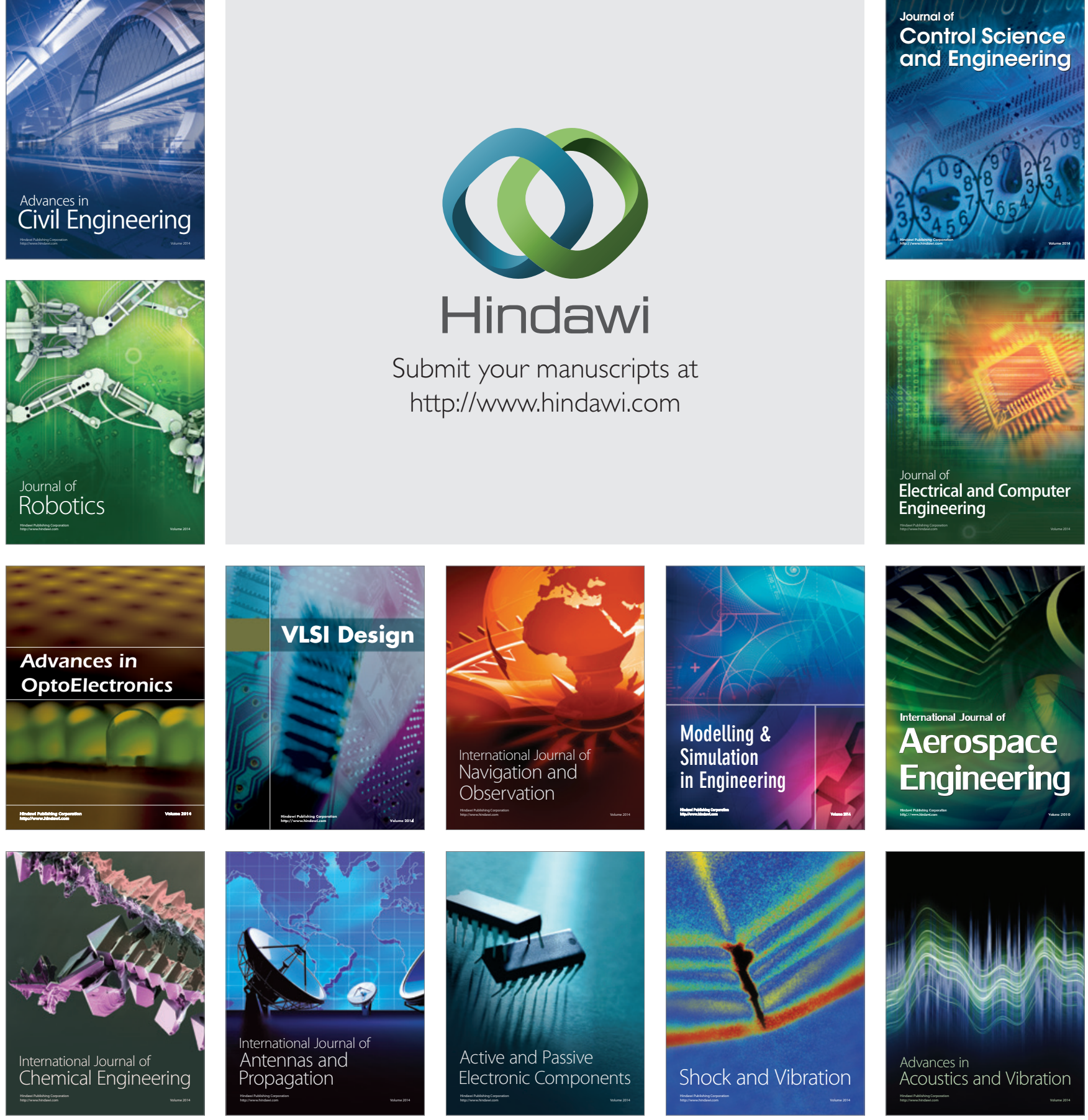